\title{
Lenalidomid reduziert das Progressionsrisiko
}

Ein verlängertes progressionsfreies Überleben und eine verlängerte Zeit bis zur Progression, das sind die Ergebnisse in den primären Endpunkten der Phase-III-Studien IFM (Intergroup Francophone du Myélome) 2005-02 und CALGB (Cancer and Leukemia Group B) 100104 zur Erhaltungstherapie mit Lenalidomid nach Hochdosischemotherapie und autologer Stammzelltransplantation (ASCT) bei Patienten mit neu diagnostiziertem multiplem Myelom (Attal M et al., ASCO 2010: \#8018; McCarthy P et al., ASCO 2010: \#8017).

In der IFM-2005-02-Studie erhielten $614 \mathrm{~Pa}$ tienten ( $<65$ Jahre) ohne Progression in den ersten 6 Monaten nach der ASCT randomisiert nach 2-monatiger Lenalidomid-Konsolidierung bis zur Progression eine Erhaltungstherapie mit Lenalidomid (10-15mg/d) oder Placebo. Die erste geplante Interimsanalyse nach median 24 Monaten Followup ergab einen Vorteil für die Lenalidomid-
Erhaltungstherapie beim progressionsfreien Überleben (Median noch nicht erreicht vs. 24 Monate mit Placebo; Hazard-Ratio [HR] 0,46; $p<0,0000001)$. Ein Vorteil für die Erhaltungstherapie mit Lenalidomid zeigte sich auch in der CALGB-100104-Studie nach median 12 Monaten Follow-up bei der Zeit bis zur Progression (Median noch nicht erreicht vs. 25,5 Monate mit Placebo; HR 0,42; $<<0,0001)$. In dieser Studie erhielten 568 Patienten $(<70$ Jahre) mit mindestens stabiler Erkrankung 90 bis 100 Tage nach der ASCT bis zur Progression Lenalidomid (10mg/d; Dosisanpassung je nach Verträglichkeit auf $5 \mathrm{mg} / \mathrm{d}$ oder $15 \mathrm{mg} / \mathrm{d}$ ) oder Placebo. In die Analyse gingen die Daten von 418 Patienten ein. Die Erhaltungstherapie wurde in beiden Studien gut vertragen. $A M$

\section{Immuntherapie bei fortgeschrittenem Melanom \\ Überlebensvorteil mit Ipilimumab}

Die Immunpotenzierung mit dem monoklonalen Antikörper Ipilimumab verlängerte in einer Phase-III-Studie das mediane Gesamtüberleben von Patienten mit fortgeschrittenem Melanom, die keine weiteren Behandlungsoptionen mehr hatten, um knapp 4 Monate. Dieser Überlebensvorteil ist unabhängig von einer zusätzlichen Peptid-Vakzinierung mit gp100.

Patienten mit metastasiertem Melanom haben aufgrund mangelnder Behandlungsoptionen eine schlechte Prognose: Das mediane Gesamtüberleben beträgt 6,2 Monate, das 1-Jahres-Überleben $25,5 \%$ und das progressionsfreie Überleben (PFS) im Median 1,7 Monate (Korn EL et al., 2008, J Clin Oncol 26: 527-534). Umso erfreulicher sind daher die Phase-III-Daten zur Immuntherapie mit Ipilimumab, sagte Steven O'Day, Los Angeles/USA. Denn mit diesem neuen Therapieansatz ist das erste Mal ein signifikanter Überlebensvorteil bei Patienten mit fortgeschrittenem Melanom erreicht worden. Der bisher noch nicht zugelassene monoklonale Antikörper Ipilimumab blockiert spezifisch das zytotoxische T-Lymphozyten-assoziierte Antigen 4 (CTLA-4), das wie eine Bremse bei der T-Zell-Aktivierung wirkt.

\section{T-Zell-Potenzierung mit Anti-CTLA-4}

In der Phase-III-Studie wurden 676 vorbehandelte Patienten mit nicht resezierbarem, metastasiertem Melanom im Stadium III und IV im Verhältnis 3:1:1 randomisiert: Ipilimumab plus Placebo ( $n=137)$, Placebo plus die experimentelle Peptidvakzine gp100 (ebenfalls zur Stimulation der T-Zell-Antwort; $n=136$ ) und Ipilimumab plus gp100 ( $n=403$; O’Day S et al., ASCO 2010: \#4; Hodi FS et al., 2010, N Engl J Med: Epub ahead of print). Primärer Endpunkt war das Gesamtüberleben.

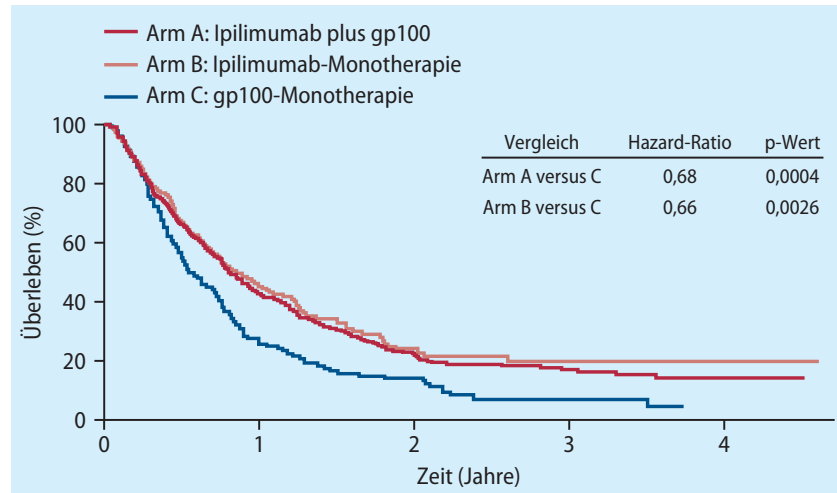

nach O'Day S et al., ASCO 2010: \#4 and oral presentation

Gesamtüberleben - Ipilimumab versus gp100 versus Kombination

Mit Ipilimumab allein überlebten die Patienten im Median 10,0 Monate, mit Ipilimumab plus gp100 10,1 Monate und mit gp100 allein 6,4 Monate. Die Überlebensraten nach 1 Jahr betrugen $46 \%, 44 \%$ und $25 \%$ und nach 2 Jahren 24\%, 22\% und 14\% (Abb.).

Wie erwartet traten unter Ipilimumab vor allem Immunreaktionen auf: Bei etwa $60 \%$ der Patienten in den Ipilimumab-Gruppen wurde ein Rash oder eine Colitis beobachtet, davon waren 14\% schwerwiegend (versus 3\% unter gp100-Monotherapie). Laut O'Day sind diese Immunreaktionen jedoch gut mit einer frühen Glucocorticoidtherapie zu handhaben.

Zurzeit wird Ipilimumab in Studien bei malignem Melanom auch als Erstlinientherapie und adjuvante Therapie geprüft sowie bei Lungen- und Prostatakarzinom. AWA 